\title{
Degeneração macular relacionada à idade: modalidades terapêticas
}

\author{
Age-related macular degeneration: therapeutic modalities
}

\begin{tabular}{lcc} 
Michel & Eid & Farah $^{1}$ \\
Akiyoshi & \multicolumn{2}{c}{ Oshima } \\
Rogério & Alves & Costa $^{3}$ \\
Jufiana & Ferraz & Sallum $^{4}$
\end{tabular}

Universidade Federal de São Paulo - Escola Paulista de Medicina - SP.

${ }^{1}$ Professor Livre Docente de Oftalmologia.

${ }^{2}$ Pós-Graduando de Oftalmologia - Doutorado.

${ }^{3}$ Pós-Graduando de Oftalmologia - Doutorado.

${ }^{4}$ Doutora em Medicina - Chefe do Setor de Retina e

Vítreo.

Endereço para correspondência: Av. Ibijaú, 331, $4^{\circ}$ Andar - São Paulo (SP) CEP 04524-020 E-mail: michel farah@uol.com.br

\section{RESUMO}

Descrever as modalidades de tratamento clínico e cirúrgico das complicações da degeneração macular relacionada à idade (DMRI).

Descritores: Fotoquimioterapia; Degeneração macular; Porfirinas; Neovascularização coroidal; Idade de início

\section{INTRODUÇÃO}

A degeneração macular relacionada à idade é responsável por perda visual grave e é a principal causa de cegueira sob o ponto de vista legal nos pacientes com mais de 50 anos de idade na maioria dos países desenvolvidos $^{(1)}$. A degeneração macular relacionada à idade é uma doença ocular degenerativa caracterizada clinicamente nas fases iniciais por alterações do epitélio pigmentado da retina e presença de drusas, sem comprometimento clinicamente significante da função visual na maioria dos casos até que ocorram formas centrais ou extensas de atrofia geográfica ou formação de neovascularização coroidiana, exsudação sub-retiniana e cicatriz fibrosa macular com baixa acentuada da visão. Ocorre um aumento da prevalência nas idades mais avançadas; assim, no estudo ${ }^{(2)}$ diagnosticou-se $1,6 \%$ de degeneração macular relacionada à idade na população entre 52 e 64 anos de idade e $27,9 \%$ em pacientes acima de 75 anos. Num estudo recentemente publicado nos EUA, a prevalência de qualquer sinal associado a degeneração macular relacionada à idade em pacientes norte-americanos maiores de 40 anos foi de 9,2\%; isto sugere que existem pelo menos 8,5 milhões de norte-americanos afetados atualmente por esta doença. Algumas diferenças raciais foram observadas, com taxas de prevalência de $9,3 \%$ entre os brancos, 7,4\% entre os negros e 7,1\% entre os americano-mexicanos ${ }^{(3)}$.

A forma seca, atrófica, geográfica ou não-neovascular, caracterizada pela presença de área atrófica, de contornos geográficos na área macular, onde se observam os grandes vasos da coróide e ausência de membrana neovascular coroidal (MNVC), ocorre na grande maioria dos casos (79\%) de degeneração macular relacionada à idade e apresenta uma evolução lenta.

Quando a membrana neovascular coroidal e/ou cicatriz disciforme acompanha essas anormalidades de fundo temos a forma "úmida", exsudativa, disciforme ou neovascular que ocorre em 15,3\% dos casos de degeneração macular relacionada à idade. Esta forma, embora tenha uma prevalência menor que a forma seca, é responsável por cerca de $80 \%$ de cegueira sob o ponto de vista legal devido à degeneração macular relacionada à idade ${ }^{(4)}$. Outro estudo mostrou que a forma neovascular é muito menos comum que a não neovascular nos adultos com idade entre 43 e 86 
anos. Na neovascularização (membrana neovascular coroidal) ocorre o crescimento de neovasos subrretinianos com vazamento de fluídos, lipídios ou lipoproteínas e sangue. Este processo leva à formação de tecido fibroso e cicatricial com desorganização da integridade estrutural da mácula e conseqüente perda de visão central ${ }^{(5)}$. Sabemos ainda que se um olho apresenta lesão subfoveal, os fatores de risco independente para o desenvolvimento de lesão exsudativa no olho adelfo são: 1 - presença de cinco ou mais drusas (risco relativo $=2,1) ; 2$ - hiperpigmentação focal (risco relativo $=2,0$ ); 3 - Presença de drusas moles $(>63 \mu \mathrm{m})$ (risco relativo $=1,5)$; 4 - Hipertensão arterial (risco relativo $=1,7$ ). A incidência de lesão exsudativa contra-lateral varia de $7 \%$ (sem nenhum dos fatores de risco) a $87 \%$ (com todos os quatro fatores de risco) em cinco anos ${ }^{(6)}$, com cerca de 4 a $12 \%$ destes olhos desenvolvendo a doença neovascular a cada ano.

Baseando-se nos achados oftalmoscópicos, biomicroscópicos e angiofluoresceinográficos, o complexo ou lesão neovascular pode apresentar três componentes: 1 - membrana neovascular coroidal clássica (áreas bem delimitadas de hiperfluorescência precoce com vazamento intenso e uniforme tardio que geralmente obscurecem as margens da lesão); 2 - membrana neovascular coroidal oculta (deslocamento do epitélio pigmentário fibrovascular e/ou vazamento tardio de origem indeterminada); 3 - fatores associados (sangue espesso, hipofluorescência por bloqueio elevada, deslocamento do epitélio pigmentário seroso). O complexo neovascular pode ainda ser classificado como bem definido uma vez que seus limites possam ser identificados em toda sua extensão $\left(360^{\circ}\right)$, independentemente do tipo angiográfico da membrana neovascular. Geralmente os complexos com componente oculto são mais difíceis de identificação, observando-se apenas um vazamento pontilhado difuso, pouco intenso(7).

Um estudo ${ }^{(8)}$ mostrou a presença e as características das membranas neovasculares anteriormente classificadas como ocultas pela angiofluoresceinografia que se apresentavam bem definidas pela videoangiografia digital com a indocianina verde. A videoangiografia com a indocianina verde facilita a identificação das membranas neovasculares coroidais ocultas, permitindo sua reclassificação em membranas neovasculares coroidais presumidas bem definidas pela indocianina verde com diâmetro menor que ou igual a um diâmetro papilar (DP). Foi introduzido o conceito de placas de neovascularização ou membrana neovascular coroidal inativa onde a área de hiperfluorescência é maior que uma área de disco em tamanho, na indocianina verde, de localização geralmente subfoveal, sendo as lesões mais comumente encontradas e apresentam menor atividade de crescimento. Os pontos ou regiões presentes de membrana neovascular coroidal ativa, também chamados focos ou pontos quentes ${ }^{(9)}$, são áreas de exsudação subrretiniana e aparecem como membrana neovascular coroidal oculta na angiofluoresceinografia e durante a indocianina verde se apresentam como lesões hiperfluorescentes, sendo geralmente observadas fora da zona foveal avascular e, portanto passíveis de tratamento. Embora a história natural da doença acabe sendo a quiescência e a interrupção do processo de neovascularização, isso ocorre normalmente às custas de fibrose subrretiniana e perda da visão central $^{(10)}$. A fotocoagulação a "laser" foi até agora o único tratamento relativamente eficaz na destruição da membrana neovascular ${ }^{(11)}$. Nos casos de membrana neovascular coroidal bem definida, extrafoveal ou justafoveal, os resultados com o tratamento por "laser" são melhores do que os do não tratamento, em relação a impedir ou retardar a perda visual ${ }^{(12-15)}$. Este tratamento baseia-se na destruição dos neovasos por fotocoagulação a "laser" de argônio ou kriptônio, não seletiva, causando danos à parte externa da retina, incluindo os fotorreceptores que estão justapostos ao epitélio pigmentado da retina e à membrana neovascular coroidal, levando à formação de cicatriz fibroglial e escotoma visual correspondente. A termofotocoagulação em pacientes com membrana neovascular coroidal subfoveal clássica, de nova lesão ou recidivante, pode levar à destruição da retina neuro-sensorial com perda visual central significante. Neste grupo, embora estudos abrangentes tenham demonstrado vantagem a partir de um ano de evolução do tratamento a "laser" em comparação à simples observação principalmente em lesões menores que duas áreas de disco (segundo o grupo de estudo Macular Photocoagulation Study (MPS Group), o benefício do tratamento é de difícil aceitação para lesões subfoveais onde é comum ocorrer perda imediata da acuidade visual ${ }^{(14)}$.

Os resultados do MPS Group mostraram que, no exame de 24 meses, os olhos tratados a "laser" perderam, em média, 3,3 linhas de acuidade visual e os não tratados, em média, 4,5 linhas. A acuidade visual média foi de 20/320 e 20/400, respectivamente. Na ocasião, a porcentagem de pacientes cuja perda foi de seis ou mais linhas de visão foi de $20 \%$ no grupo tratado e $37 \%$ no grupo não tratado ${ }^{(16)}$.

Tratamento preventivo com fotocoagulação a "laser" nas drusas moles tem surgido como uma nova opção terapêutica da degeneração macular relacionada à idade no sentido de se prevenir o aparecimento de membrana neovascular coroidal e tem sido avaliado numa série de ensaios clínicos ${ }^{(17-21)}$. Apesar dos resultados promissores na sua fase inicial em reabsorver as drusas moles com a fotocoagulação a "laser", parece que esse tratamento não impede o aparecimento da membrana neovascular coroidal no decorrer de alguns anos, como foi observado no ensaio multicêntrico randomizado (Choroidal Neovascularization Prevention Trial) que mostrou uma incidência aumentada de membrana neovascular coroidal em curto prazo nesses olhos de alto risco ${ }^{(22-23)}$.

Cirurgia submacular para retirada da membrana neovascular coroidal tem sido utilizada para outras doenças relacionadas ao desenvolvimento de membrana neovascular coroidal, como na síndrome da histoplasmose ocular ${ }^{(24-25)}$. Teoricamente a remoção cirúrgica da membrana neovascular coroidal pode impedir o alargamento do defeito visual, limitar a perda da visão central e permitir que os fotorreceptores adjacentes à 
membrana neovascular coroidal voltem a funcionar normalmente. A cirurgia também pode facilitar a decisão quanto à conduta a ser tomada frente à membrana neovascular coroidal associada a grandes hemorragias subfoveais. As séries de casos que têm sido apresentadas têm a técnica cirúrgica bem detalhada e período de seguimento relativamente curto, de 12 meses ou menos, além de faltar grupo controle. Existe ainda uma alta taxa de recidivas (até $46 \%$ ) e complicações tais como aceleração na opacificação do cristalino, desenvolvimento de roturas retinianas, assim como descolamentos de retina e coróide. Um grande ensaio clínico, multicêntrico e randomizado em 11 centros nos EUA está atualmente sendo realizado (Submacular Surgery Trials Pilot Study, com suporte do National Eye Institute dos National Institutes of Health) e irá revelar o papel real da cirurgia submacular no tratamento da membrana neovascular coroidal pela degeneração macular relacionada à idade. Outras modalidades de tratamento cirúrgico estão sendo investigadas, tais como translocação de mácula, transplante do epitélio pigmentado da retina e epitélio iriano autólogo na mácula ${ }^{(26-27)}$.

Radioterapia tem sido proposta para impedir a proliferação de células endoteliais de capilares subrretinianos recémformados e para induzir a obliteração de neovasos na membrana neovascular coroidal. Os investigadores de estudos pilotos avaliando os efeitos da terapia por radiação concluíram que os resultados eram promissores, embora as lesões fossem geralmente grandes, de limites pouco precisos, com visão bastante baixa e que nem sempre tinham um componente clássico da $\mathrm{MNVC}$, fatores que poderiam ter um impacto nos resultados aparentemente favoráveis comparados com os dos ensaios do MPS. Num estudo multicêntrico, não randomizado ${ }^{(28)}$, revendo 111 casos de pacientes submetidos a feixe de radiação externa, mostraram que o tratamento falhou em controlar ou induzir regressão de membrana neovascular coroidal ativa. Devido a isso, novos ensaios clínicos estão em andamento nos EUA para avaliar a eficácia da radioterapia no tratamento da degeneração macular relacionada à idade, forma exsudativa.

As drogas anti-angiogênicas ocupam um lugar promissor no tratamento das membranas neovasculares da degeneração macular relacionada à idade, pois apresentam o potencial de evitar os efeitos danosos provocados pela fotocoagulação no tecido macular, tratar lesões mal definidas ou lesões com maior componente oculto do que clássico, diminuir a taxa de recorrência pós-tratamento com "laser" convencional e ainda impedir que olhos com degeneração macular não neovascular relacionada à idade evoluam para a forma exsudativa. Alfa-2 Interferon tem se mostrado eficaz como droga anti-angiogênica com base em trabalhos realizados em ciência básica, estudos em neovascularização experimental em macacos e em diversas doenças sistêmicas em humanos. Resultados conflitantes de vários estudos pilotos têm sido publicados considerando sua eficácia na degeneração macular relacionada à idade ${ }^{(29)}$. Todos esses estudos apresentaram amostras pequenas e a maioria deles não foi randomizada para tratamento ou observação. Recentemente, foi realizado um ensaio clínico multicêntrico, randomizado que tinha recrutado pacientes com membrana neovascular coroidal subfoveal em que os mesmos foram divididos em quatro grupos, recebendo 1,5 - 3 - 6 milhões UI (Unidade Internacional) ou placebo, administrados três vezes por semana por 12 meses; 479 olhos foram recrutados em 53 centros, incluindo a USP e a UNIFESP/EPM. Ao final de 12 meses após a entrada no estudo o tratamento não se mostrou benéfico e possivelmente prejudicial ${ }^{(30)}$. Porém, esses resultados serviram para mostrar o curso natural bastante variável da membrana neovascular coroidal na degeneração macular relacionada à idade, especialmente dentro de um ano após a sua apresentação inicial. Essa variável levou a conclusões errôneas de que esse tratamento fosse benéfico, sendo que tais casos não têm uma deterioração significante num seguimento curto e que poderiam ter tido o mesmo resultado sem tratamento. Não obstante, dado o impacto de perda visual devida à degeneração macular relacionada à idade, estudos continuam em animais e no homem para investigar o benefício potencial de outros agentes farmacológicos tais como os inibidores do fator vascular de crescimento endotelial, talidomida, inibidores da matriz metaloproteinase, esteróides angiotáxicos, angiostatina, endostatina, que apresentam efeitos anti-angiogênicos e têm o potencial de bloquear a progressão da neovascularização.

Termoterapia transpupilar é uma técnica de laser em que o calor é liberado lentamente para a coróide e para o epitélio pigmentado da retina através da pupila, utilizando um "laser" de diodo de $810 \mathrm{~nm}$, modificado para ter uma mira de tamanho grande e tempo prolongado para obter uma hipertermia localizada e profunda. Esta técnica de "laser" tem sido utilizada para o tratamento de pequenos melanomas de coróide e está sendo utilizada atualmente como uma nova modalidade de tratamento para membranas neovasculares coroidais subfoveais ocultas. Resultados preliminares mostraram que a termoterapia transpupilar não apresentou efeitos colaterais e pode ser eficaz em tratar membranas neovasculares coroidais ocultas na degeneração macular relacionada à idade. A termoterapia transpupilar é possibilitada de uma lâmpada de fenda utilizando um feixe ajustável de 1,2 $\mathrm{mm}, 2,0 \mathrm{~mm}$ ou 3,0 $\mathrm{mm}$. Novos modelos com miras maiores estão sendo elaborados pela UNIFESP/EPM em parceria com a Opto Eletrônica/Brasil. O tratamento é feito com uma mira que englobe toda a lesão. Os parâmetros utilizados do "laser" são: duração de 60 segundos e potência entre 250 e $1000 \mathrm{~mW}$, dependendo do tamanho da mira. Geralmente, para uma mira de $3 \mathrm{~mm}$, a potência inicial é de $800 \mathrm{~mW}$. Tipicamente, nenhuma mudança na coloração é observada no final do tratamento. Em contraste ao "laser" convencional, o tratamento com hipertermia minimiza a coagulação tecidual a tecidos vizinhos; privilegiando a penetração profunda na coróide e no epitélio pigmentado da retina. A irradiação infravermelha é benéfica porque a penetração tecidual é alta e a absorção pelos meios oculares é minimizada. 
A grande mira utilizada nesta técnica é vantajosa porque grandes áreas de membrana neovascular coroidal oculta podem ser tratadas mais uniformemente e o diâmetro aumentado do feixe de irradiação permite uma dissipação mínima de calor para os tecidos vizinhos. Nenhum paciente apresentou perda de visão aguda imediatamente após o tratamento e nenhuma outra complicação foi observada. No estudo piloto a estabilização da visão ocorreu em $77 \%$ dos pacientes e diminuição da exsudação foi observada em $94 \%^{(31)}$.

Uma outra modalidade de tratamento para membrana neovascular coroidal por degeneração macular relacionada à idade que está sendo investigada é o tratamento do vaso nutridor usando angiografia com indocianina verde Phi-Motion, de alta velocidade, para identificar o vaso que penetra na área da membrana neovascular coroidal e tratá-lo com "laser" infravermelho de diodo de $810 \mathrm{~nm}$, intensificado pela indocianina verde. O tratamento a "laser" a um simples vaso nutridor pode parar a exsudação de uma membrana neovascular coroidal clássica ou oculta e os efeitos positivos podem ser rápidos, resultando em melhora da acuidade visual, fluído subrretiniano e diminuição do vazamento determinado angiograficamente dentro de algumas horas. Apesar do curto período de seguimento (seis meses), a taxa de recidiva de $27 \%$ foi baixa em relação às outras modalidades ${ }^{(32)}$.

\section{TERAPIA FOTODINÂMICA}

As estratégias em desenvolvimento visaram dano mais seletivo aos neovasos com preservação da retina neuro-sensorial que reveste a lesão. Uma dessas estratégias é a terapia fotodinâmica com a verteporfirina, que se baseia na exposição dos tecidos tratados com fotossensibilizantes à luz de baixa intensidade para provocar efeitos fotoquímicos. A terapia fotodinâmica é um processo de duas etapas: 1) administração intravenosa do fotossensibilizante e 2) irradiação da luz ${ }^{(33)}$. Os corantes fotossensibilizantes ficam retidos preferencialmente em tumores, sobretudo no tecido neovascular do tumor, o que permite o tratamento seletivo desse tecido patológico ${ }^{(34)}$. O corante é ativado no estado triplo após exposição à luz em comprimento de onda de $689 \mathrm{~nm}$ e interage com o oxigênio e outros compostos formando intermediários reativos, como o oxigênio singleto, que podem então provocar desorganização das estruturas celulares ${ }^{(35)}$. Entre os possíveis alvos celulares estão a membrana celular, a mitocôndria, as membranas lisossomais e o núcleo. As evidências dos modelos de tumor e de neovasculatura indicam que a oclusão da vasculatura é um importante mecanismo de terapia fotodinâmica, que ocorre por dano às células endoteliais, com subseqüente adesão e desgranulação plaquetária e formação de trombo. Existem evidências cada vez maiores de que a terapia fotodinâmica cause morte do tumor pela oclusão dos vasos que o alimentam, bem como por efeito citotóxico direto sobre suas células. Esse mecanismo despertou o interesse em utilizar a terapia fotodinâmica no tratamento de doenças oftalmológicas caracterizadas por neovascularização.

Os estudos anteriores da terapia fotodinâmica utilizaram fotossensibilizantes mais antigos, como o derivado da hematoporfirina ou o rosa-bengala, e foram limitados pela capacidade fotossensibilizante mais fraca ou pela fotossensibilidade cutânea prolongada. Os fotossensibilizantes mais recentes foram desenvolvidos para superar essas dificuldades. Entre eles está a verteporfirina ${ }^{(36)} \mathrm{e}$ a indocianina verde ${ }^{(42)}$.

\section{A) Verteporfirina}

A verteporfirina é um fotossensibilizante potente composto de dois regioisômeros com propriedades antineoplásticas e imunomodulatórias, bem como ao seu potencial de estabilização da visão na degeneração macular relacionada à idade, forma neovascular.

A verteporfirina é administrada por via endovenosa em formulação com base lipídica. Logo após a infusão, a verteporfirina distribui-se na fase lipoprotêica, particularmente na fração Low Density Lipoprotein (LDL). Demonstrou-se que os tecidos neoplásicos e a neovascularização dentro dos tumores aumentam o número de receptores de LDL e acredita-se que esse seja um mecanismo importante de aumento da seletividade da verteporfirina por esses tecidos. Os valores médios da meia-vida de eliminação plasmática terminal variam de aproximadamente 5 a 6 horas para os dois regioisômeros da verteporfirina. A depuração ocorre principalmente pela bile e fezes $(90 \%)$, sendo menos de $1 \%$ depurado pelos rins e urina $^{(37)}$.

Os estudos das fases I/II mostraram que uma única sessão de terapia fotodinâmica com a verteporfirina pode estabilizar de forma segura a extensão de vazamento das lesões de neovascularização em pacientes com degeneração macular relacionada à idade. No total, 609 pacientes foram randomizados para tratamento, dos quais aproximadamente $2 / 3$ receberam terapia fotodinâmica ativa com a verteporfirina (402 pacientes). Após seguimento por 12 meses os resultados mostraram que os pacientes tratados apresentaram melhora da acuidade visual comparados com o grupo placebo (61\% versus $46 \%$ no grupo placebo, diferença de $15 \%$ a favor da verteporfirina, $p<0,001)$. A análise do subgrupo de pacientes com lesões predominantemente clássicas apresentou taxa favorável de $67 \%$ para verteporfirina versus $39 \%$ para o placebo $(\mathrm{p}<0,001)$. Resultados semelhantes foram obtidos após seguimento por dois anos ${ }^{(38)}$.

Com base no conhecimento acumulado até o momento ${ }^{(39)}$, a terapia fotodinâmica com a verteporfirina representa um avanço real em relação à termofotocoagulação a "laser" no tratamento da neovascularização subfoveal, uma vez que oclui seletivamente a membrana neovascular sem destruir a retina neuro-sensorial sobrejacente à lesão. Além disso, a terapia com verteporfirina também pode ser utilizada para tratar grandes lesões de neovascularização sem apresentar os efeitos deletérios à visão em comparação ao não tratamento. É 
mais freqüente os pacientes tratados com a verteporfirina, por pelo menos dois anos, apresentarem estabilidade ou melhora da visão relativamente aos não tratados ${ }^{(40-41)}$.

\section{B) Indocianina verde}

Um estudo experimental recente demonstrou que a indocianina verde (ICG) é um fotossensibilizante eficaz na oclusão fotodinâmica da coriocapilar normal de coelhos pigmentados. O tratamento da coriocapilar somente com o laser de diodo, de $810 \mathrm{~nm}$, sem o fotossensibilizante, utilizando irradiâncias baixas, não resultou em qualquer dano retiniano ou coroidal, demonstrado histológica e fluoresceinograficamente. Usando ICG, a vasculatura subrretiniana foi efetivamente ocluída, com mínimo dano à retina neuro-sensorial adjacente, na dose de irradiação de $6,3 \mathrm{~J} / \mathrm{cm}^{2}$. Efeitos coroidais mais intensos foram observados com doses mais elevadas de luz. Exame clínico e retinografias de fundo não revelaram mudança na coloração retiniana após a TFD, embora um leve esbranquecimento retiniano tenha sido observado no dia seguinte ${ }^{(42)}$.

\section{A B S T RAC T}

To describe the clinical and surgical therapeutic modalities in age-related macular degeneration (ARMD).

Keywords: Photochemoterapy; Macular degeneration; Porphyrins; Choroidal neovascularization; Age of onset

\section{REFERÊNCIAS}

1. Ferris FL, Fine SL, Hyman L. Age-related macular degeneration and blindness due to neovascular maculopathy. Arch Ophthalmol 1984;102:1640-2.

2. Leibowitz HM, Krueger DE, Maunder LR, Milton RC, Kini MM, Kahn HA et al. The Framingham Eye Study monograph: An ophthalmological and eidemiological study of cataract, glaucoma, diabetic retinopathy, macular degeneration, and visual acuity in a general population of 2631 adults, 19731975. Surv Ophthalmol 1980;24(Suppl):335-610.

3. Klein R, Rowland ML, Harris MI. Racial/ethnic differences in age-related maculopathy. Third National Health and Nutrition Examination Survey. Ophthalmology 1995;102:371-81.

4. Chandra SR, Gragoudas ES, Friedman E, Van Buskirk EM, Klein ML. Natural history of disciform degeneration of the macula. Am J Ophthalmol 1974;78:579-82.

5. Macular Photocoagulation Study Group. Laser photocoagulation of subfoveal neovascular lesions of age-related macular degeneration. Updated findings from two clinical trials. Arch Ophthalmol 1993;111:1200-9.

6. Bressler SB, Maguire MG, Bressler NM, Fine SL. Relationship of drusen and abnormalities of the retinal pigment epithelium to the prognosis of neovascular macular degeneration. The Macular Photocoagulation Study Group. Arch Ophthalmol 1990;108:1442-7.

7. Stevens TS, Bressler NM, Maguire MG, Bressler SB, Fine SL, Alexander J et al. Occult choroidal neovascularization in age-related macular degeneration. A natural history study. Arch Ophthalmol 1997;115:345-50.

8. Yannuzzi LA, Slakter JS, Sorenson JA, Guyer DR, Orlock DA. Digital indocyanine green videoangiography and choroidal neovascularization. Retina 1992;12:191-223.

9. Gass JD, Pathogenesis of disciform detachment of the neuroepithelium: III. Senile disciform macular degeneration. Am J Ophthalmol 1967;63:(Pt2):617-44.

10. Macular Photocoagulation Study Group. Recurrent choroidal neovascularization after argon laser photocoagulation for neovascular maculopathy. Arch Ophthalmol 1986;104:503-12.
11. Argon laser photocoagulation for senile macular degeneration. Results of a randomized clinical trial. Arch Ophthalmol 1982;100:912-8.

12. Macular Photocoagulation Study Group. Krypton laser photocoagulation for neovascular lesions of age-related macular degeneration. Results of a randomized clinical trial. Arch Ophthalmol 1990;108:816-24.

13. Macular Photocoagulation Study Group. Laser photocoagulation of subfoveal neovascular lesions in age-related macular degeneration. Results of a randomized clinical trial. [Comment on: Arch Ophthalmol 1994 112:874-5]. Arch Ophthalmol 1991;109:1220-31.

14. Macular Photocoagulation Study Group. Influence of treatment extent on the visual acuity of eyes treated with Krypton laser for juxtafoveal choroidal neovascularization. Arch Ophthalmol 1995;113:190-4.

15. Macular Photocoagulation Study Group. Argon laser photocoagulation for neovascular maculopathy. Five-year results from randomized clinical trials. Arch Ophthalmol 1991;109:1109-14.

16. Wetzig PC. Treatment of drusen-related aging macular degeneration by photocoagulation. Trans Am Ophthalmol Soc 1988:276-90.

17. Sigelman J. Foveal drusen resorption one year after perifoveal laser photocoagulation. Ophthalmology 1991;98:1379-83.

18. Figueroa MS, Regueras A, Bertrand J. Laser photocoagulation to treat macular soft drusen in age-related macular degeneration. Retina 1994;14:391-6.

19. Frennesson IC, Nilsson SE. Effects of argon (green) laser treatment of soft drusen in early age-related maculopathy: a 6 month prospective study. Br J Ophthalmol 1995;79:905-9.

20. Little HL, Showman JM, Brown BW. A pilot randomized controlled study on the effect of laser photocoagulation of confluent soft macular drusen. Ophthalmology 1997; 104:623-31.

21. Sandberg MA, Weiner A, Miller S, Gaudio AR. High-risk characteristics of fellow eyes of patients with unilateral neovascular age-related macular degeneration. Ophthalmology 1998;105:441-7.

22. Choroidal Neovascularization Prevention Trial Research Group. Laser treatment in eyes with large drusen. Short-term effects seen in a pilot randomized clinical trial. Ophthalmology 1998;105:11-23.

23. Thomas MA, Grand MG, Williams DF, Lee CM, Pesin SR, Lowe MA. Surgical management of subfoveal choroidal neovascularization. Ophthalmology 1992; 99:952-68; discussion p.975-6.

24. Berger AS, Conway M, Del Priore LV, Walker RS, Pollack JS, Kaplan HJ. Submacular surgery for subfoveal choroidal neovascular membranes in patients with presumed ocular histoplasmosis. Arch Ophthalmol 1997;115:991-6.

25. Peyman GA, Blinder KJ, Paris CL, Alturki W, Nelson NC, Desai U. A technique for retinal pigment epithelium transplantation for age-related macular degeneration secondary to extensive subfoveal scarring. Ophthalmic Surg 1991;22:102-8.

26. De Juan E, Loewenstein A, Bressler NM, Alexander J. Translocation of the retina for management of subfoveal choroidal neovascularization II: a preliminary report in humans. Am J Ophthalmol 1998;125:635-46.

27. Spaide RF, Guyer DR, McCormick B, Yannuzzi LA, Burke K, Meldelsohn $\mathrm{M}$, et al. External beam radiation therapy for choroidal neovascularization. Ophthalmology 1998;105:24-30.

28. Stalmans P, Leys A, Limbergen E. External beam radiotherapy (20 Gy-2Gy fractions) fails to control the growth of choroidal neovascularization in agerelated macular degeneration: A review of 111 cases. Retina 1997;17:481-92.

29. Miller JW, Stinson W, Folkman J. Regression of iris neovascularization with systemic alpha-interferon. Ophthalmology 1993;100:9-14.

30. Pharmacological therapy for macular degeneration study group. Interferon alpha 2-a is ineffective for patients with choroidal neovascularization secondary to age-related macular degeneration. Arch Ophthalmol 1997;115:865-72.

31. Reichel E, Berrocal AM, Ip M, Kroll AJ, Desai V, Duker JS et al. Transpupillary thermotherapy of occult subfoveal choroidal neovascularization in patients with age-related macular degeneration. Ophthalmology 1999;106: 1908-14.

32. Flower RW, Glaser BM,, Murphy RP. Experimental studies of indocianine green dye-enhanced photocoagulation of choroidal neovascularization feeder vessels. Am. J. Ophthalmol. 2000;129(4):501-12.

33. Gomer CJ. Photodynamic therapy in the treatment of malignancies. Semin Hematol 1989;26:27-34.

34. Roberts WG, Hasan T. Role of neovasculature and vascular permeability on the tumor retention of photodynamic agents. Cancer Res 1992;52:924-30.

35. Kreimer-Birnbaum M. Modified porphyrins, chlorins, phthalocyanines, and purpurins: second-generation photosensitizers for photodynamic therapy. Semin Hematol 1989;26:157-73.

36. Richter AM, Waterfield E, Jain AK, Allison B, Sternberg ED, Dolphin D et 
al. Photosensitising potency of structural analogues of benzoporphyrin derivative (BPD) in a mouse tumour model. Br J Cancer 1991;63:87-93.

37. Role of delivery vehicles for photosensitizers in photodynamic therapy of tumors. J Photochem Photobiol B: Biol 1997;37:189-95.

38. Treatment of Age-related macular degeneration with Photodynamic Therapy (TAP) Study Group. Verteporfin (Visudyne TM) therapy of subfoveal CNV in age-related macular degeneration. One year results of two randomized clinical trial - TAP report \# 1. Arch Ophthalmol 1999;117:1329-45.

39. Lucena EG, Miller JW. Benzopophyrin (Verteporfin) Photodynamic Therapy for Choroidal Neovascularization in Age-related Macular Degeneration. Arq Bras Oftalmol 2000;63:511-18.
40. Schmidt-Erfurth U, Miller JW, Sickenberg M, Laqua H, Barbazetto I, Gragoudas ES et al. Photodynamic therapy with verteporfin for choroidal neovascularization caused by age-related macular degeneration: results of retreatments in a phase 1 and 2 study. Arch Ophthalmol 1999;117:1177-87.

41. Miller JW, Schmidt-Erfurth U, Sickenberg M, Pournaras CJ, Laqua H, Barbazetto I et al. Photodynamic therapy with verteporfin for choroidal neovascularization caused by age-related macular degeneration: results of a single treatment in a phase 1 and 2 study. Arch Ophthalmol 1999;117:1161-73.

42. Costa, R.A.; Farah, M.E.; Freymüller, E.; Morales, P.H.; Smith, R.; Cardillo, J.A. Choriocapillaris photodynamic therapy using indocynine green. AJO. No prelo 2001.

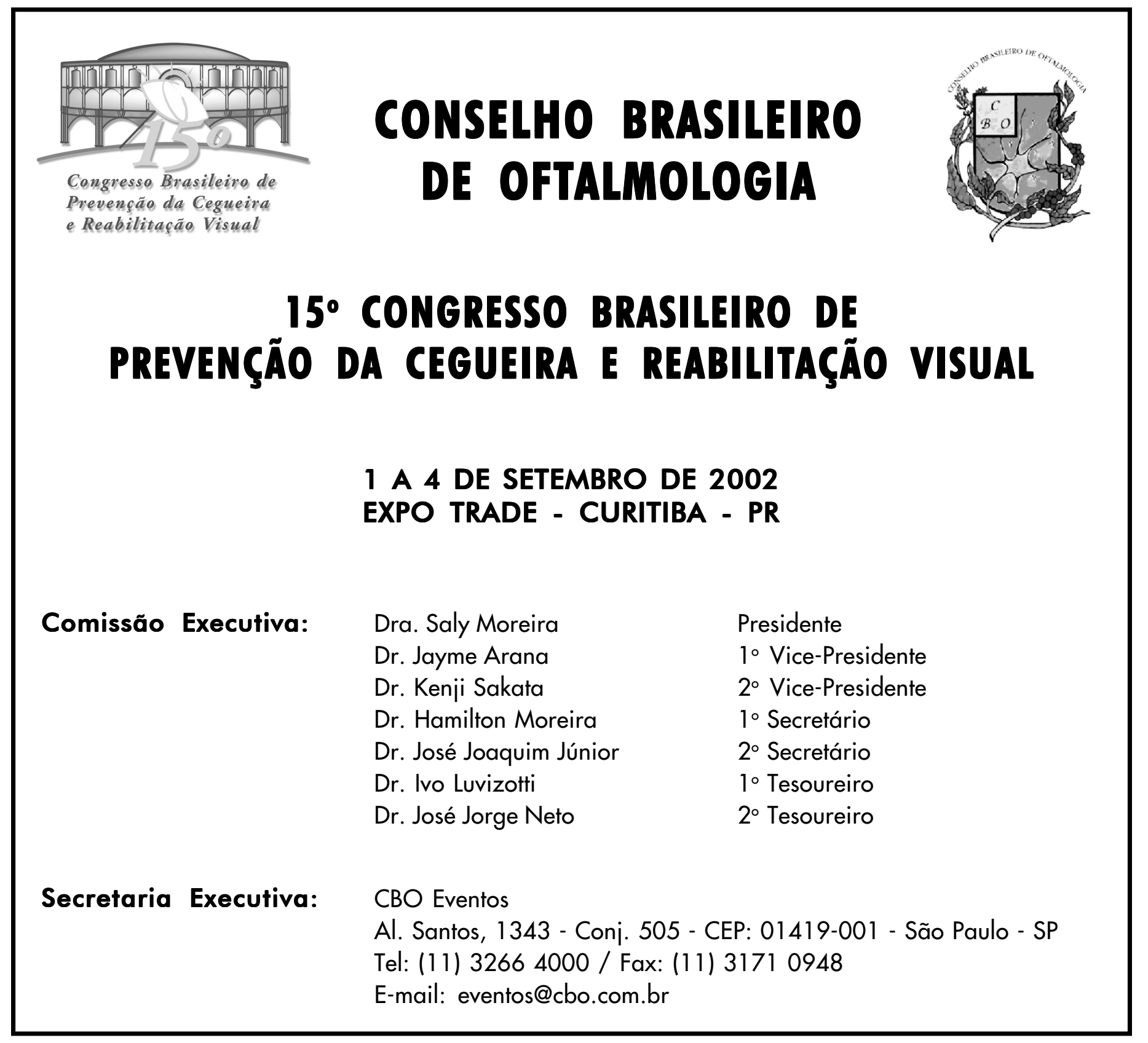

